\title{
Control of Three- and Four-Joint Arm Movement: Strategies for a Manipulator With Redundant Degrees of Freedom
}

\author{
H. Cruse \\ M. Brüwer \\ J. Dean \\ Department of Biological Cybernetics \\ University of Bielefeld \\ Germany
}

\begin{abstract}
Control of arm movements when the number of joints exceeds the degrees of freedom necessary for the task requires a strategy for selecting specific arm configurations out of an infinite number of possibilities. This report reviews strategies used by human subjects to control the shoulder, elbow, and wrist (three degrees of freedom) while moving a pointer to positions in a horizontal plane (two degrees of freedom). Analysis of final arm configurations assumed when the pointer was at the target showed the following: (a) Final arm configurations were virtually independent of the configuration at the start of the pointing movement, (b) subjects avoided configurations subjectively felt to be uncomfortable (e.g., those with extreme flexion or extension of the wrist), and (c) the results could be simulated by assigning hypothetical cost functions to cach joint and selecting the arm configuration that minimized the sum of the costs. The fitted cost functions qualitatively agreed with psychophysically determined comfort; they appeared to depend on joint angle and on muscular effort. Simple neural networks can learn implicit representations of these cost functions and use them to specify final arm configurations. The minimum cost principle can be extended to movements that use the fingers as a fourth movable segment. For this condition, however, experiments showed that final configurations of the arm depended upon initial configurations. Analysis of movement trajectories for arms with three degrees of freedom led to a control model in which the minimum cost principle is augmented by a mechanism that distributes required joint movements economically among the three joints and a mechanism that implements a degree of mass-spring control.
\end{abstract}

Key words; arm, cost function, joint control, neural nets

$\mathbf{T}$ he primary task for a limb of an animal or a robot usually can be defined as movement of the end effector, be it hand, foot, or some mechanical device, to particular positions or along particular trajectories in the work space. The control problem on the kinematic level is to find suitable joint angles to move the end effector to the desired position. In solving this problem, one important feature is whether the arm has redundant degrees of freedom. If the manipulator has exactly as many joints as are necessary to solve a given task, there are no redundant degrees of freedom, and the solution to the task specifies one and only one limb configuration. In the redundant case, the manipulator has more joints than are strictly needed, so a given task can be performed by using many different combinations of joint angles. The simplest task, placing the end point of the arm at a particular position, can be performed with an infinite number of possible configurations. Thus, one important question is how the control system selects one configuration out of all the possible configurations. To choose a definite combination of joint angles for a redundant system, we need additional constraints. These constraints effectively decrease the degrees of freedom in such an "underconstrained" system.

In this article, experiments are reviewed that used the control of the human arm as an example to address this problem. Experimental data were collected from human subjects making simple target movements by moving a pointer in a horizontal plane on the surface of a table. In most experiments, three joints (shoulder, elbow, and wrist) were allowed to move, but the arm was constrained to lie in the horizontal plane through the shoulder joint, and the palm was held vertically. Thus, the movements involved three revolute joints with vertical axes of rotation. In the initial experiments on static arm configurations, arm posi-

Correspondence address: Holk Cruse, Department of Biological Cybernetics, Faculty of Biology, University of Bielefeld, P.O. Box 100131, D-33501, Bielefeld, Germany. 
tion was recorded on videotape and measured from a video monitor. In subsequent experiments, joint angles were measured with a kind of goniometer. The arm of the subject was restrained on an artificial arm (manipulandum) that was free to glide over a horizontal plane. The manipulandum was adjusted so that the axes of rotation of the three joints of the human subject and those of the artificial arm were aligned. The pointer was the distal segment of the manipulandum, corresponding to the hand. It extended the line of the hand by $28 \mathrm{~cm}$, so that the hand plus the pointer had about the same length as the upper and the lower arm segments. The joints of the artificial arm carried potentiometers to measure the values of the joint angles.

The work space, the set of all positions reachable by the tip of the pointer, was defined by two cartesian coordinates $(x, y)$. Therefore, only two joints were required to move the end effector to any point in most of the work space. The existence of the third joint introduced an additional degree of freedom, making the system redundant. The objective of the research was to define the nature of the constraints in this three-joint system. The following discussion considers first the static problem, that is, what the resting arm configuration is for each position of the pointer, and then the kinematic problem, namely, how movements are performed. Finally, some preliminary experiments with four-joint arm movements will be described.

\section{Static Control: Selection of Arm Configurations in Pointing to a Target}

In the first series of experiments, the subject was asked to move the tip of the pointer from different starting points to each of 20 target points. After the pointer reached the target, the joint angles were recorded.

The results obtained from the four subjects differed considerably; Figure 1 illustrates the two extremes. Thus, the question arises whether each subject used a qualitatively different strategy or whether these different results are consistent with a common strategy. The second hypothesis was supported by a model calculation based upon hypothesized cost functions. This calculation is done as follows: Each joint is assigned a cost function that defines a cost value for each joint angle. The cost functions have a minimum near the middle of the range of joint movement; they increase toward either of the extreme angles. The total cost of an arm configuration is defined as the sum of the cost values for all joints. According to this hypothesis, the arm configuration for pointing to a given target in the work space is the one with the minimum total cost of the geometrically possible configurations (minimum cost principle). In this way, the effective number of degrees of freedom of the system is reduced and the redundancy problem can be solved. Moreover, the cost functions also serve to keep the joint angles away from the extremes (Cruse, 1986). It should be stressed that these cost functions are abstract entities. They may be purely descriptive and need not have a physiological

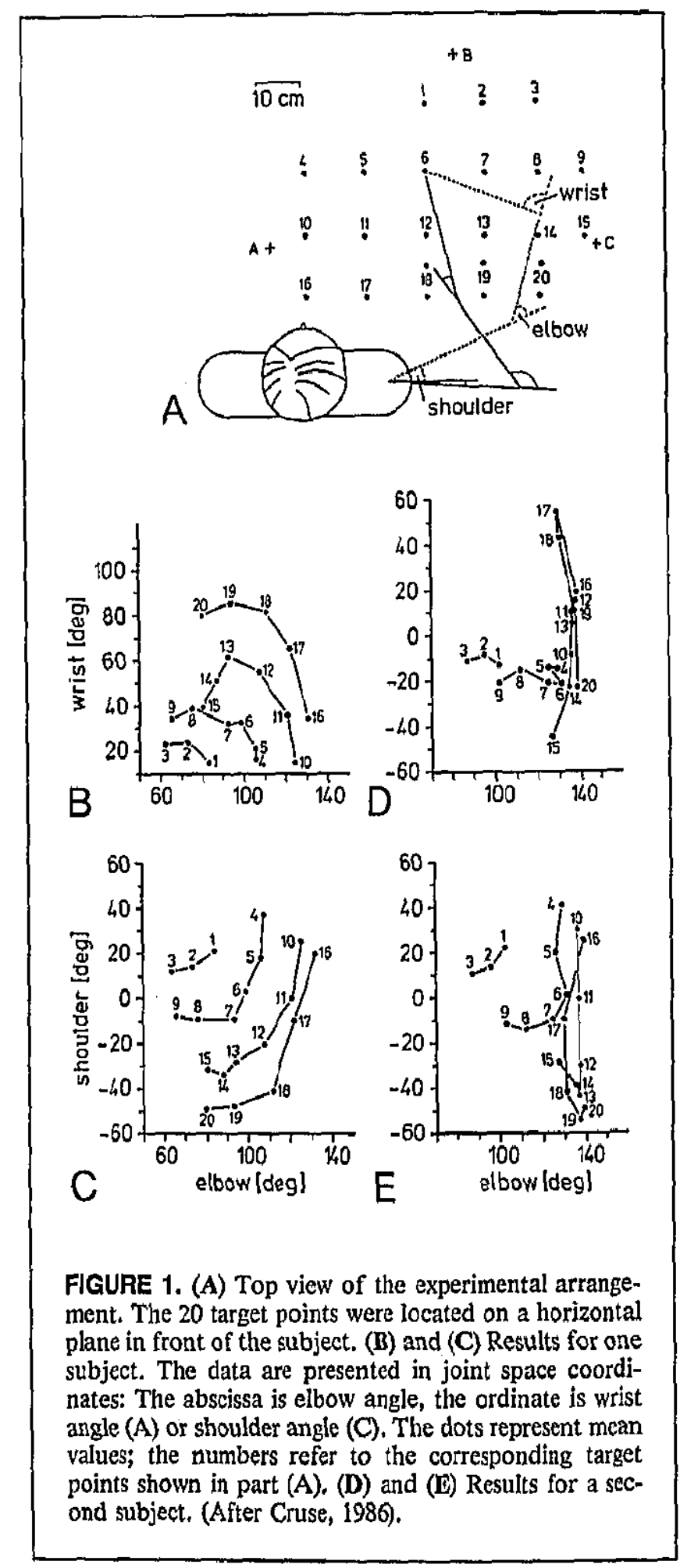

correlate. A similar approach to joint control was proposed by Rosenbaum, Slotta, Vaughan, and Plamondon (1991).

The next step was to describe these hypothetical cost functions quantitatively. In a second series of experiments, each of five subjects performed movements to 40 target points, as described above. Then for each subject, cost functions for each joint were fitted to the data, in the sense of minimizing the mean square deviation between calculated and measured angles. Qualitatively, the cost functions were assumed to be U-shaped but not necessarily symme- 

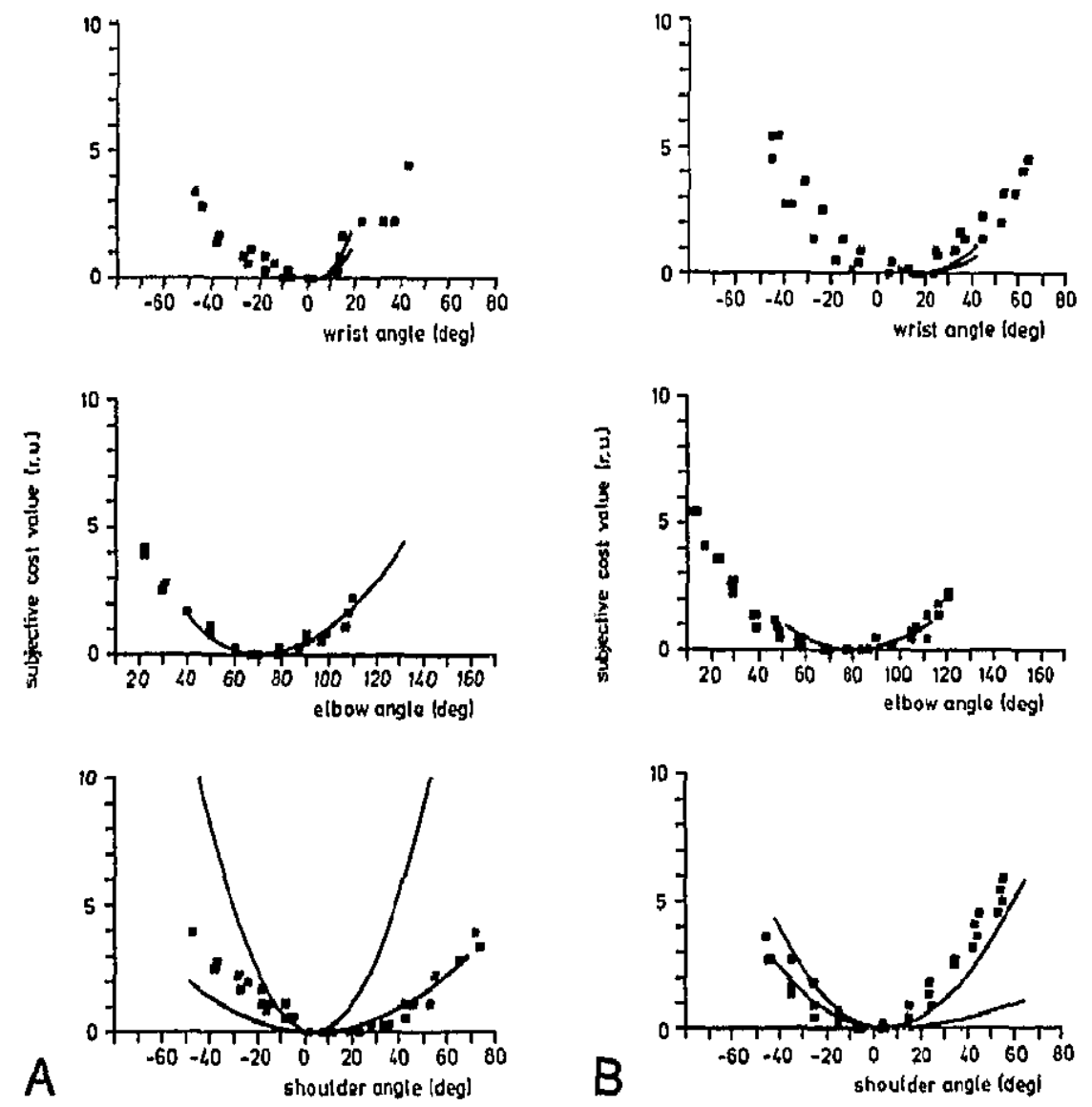

FIGURE 2. Comparison of psychophysically measured cost or "comfort" functions with hypothetical cost functions derived from the arm configuration used in pointing. Data are illustrated for two subjects (A), (B). The points represent the subjective discomfort in relative units (r.u.), measured separately for each joint. For the elbow, the solid lines represent the two parabola branches that gave the best approximation to these psychophysical results. For shoulder and wrist, see text for explanation. The parabolas were plotted only for the range of angles that actually was used in pointing (Cruse et al., 1990).

tric about the minimum. Quantitatively, they were assumed to consist of two parabolic branches that could have different slopes. Such functions can be specified by three parameters: one for the location of the minimum and two for the coefficients of the two branches. The parameters were obtained by approximation, using simulated annealing (Kirkpatrick, Gelatt, \& Vecchi, 1983).

Preliminary calculations showed that allowing all nine parameters (three for each of the three cost functions) to vary freely led to a considerable variability in the fitted values. Significant correlations between some parameters in repeated fits indicated that some of the variability was a matter of arbitrary scaling or redundancy, however. Fitting all nine parameters, therefore, contains superfluous degrees of freedom. To obtain more constant results, we repeated the approximation with the parameters of the elbow cost function set to those values that were obtained for psychophysically measured cost functions (see below). Thus only six parameters were fitted. To obtain a measure for the mean value and the standard deviation of these six param- eters for each subject, we repeated the fitting procedure 10 times. The solid lines in Figure 2 show the parabola branches that represent the $\pm 1 S D$ range obtained by these calculations for shoulder and wrist.

Expressed in terms of the joint angles as the mean standard deviation of the difference between fitted and measured angles (mean over all joints and all target positions), the variation in the fit was equivalent to \pm 4.1 and $4.0^{\circ}$ for the two subjects shown in Figure 2. The corresponding mean standard deviations for the angles measured in the targeting experiments were typically $\pm 2.9^{\circ}$. Results of another three subjects were presented in Cruse, Wischmeyer, Brïwer, Brockfeld, and Dress (1990).

These results supported the assumption that the arm position at the end of a movement can be described on the basis of cost functions. It was then of interest to examine whether a psychophysical correlate of the hypothetical cost functions can be found. To this end, psychophysical investigations were carried out to measure the subjective comfort of the different joint angles. Each subject was first asked to 
find the arm position that was most comfortable. This is called the minimum position. Then the joint to be tested was placed by the experimenter in a new angle, and the subject was asked to assign a number to the subjective feeling of comfort. Five subjects were tested. Two examples are shown by the squares in Figure 2. They show U-shaped curves that can be approximated by parabola branches. In general, the two branches of the parabola have different slopes. A comparison between the comfort functions measured by the psychophysical experiments and the hypothetical cost functions derived from the freely selected arm configurations showed the following: The positions of the minima agreed quite well. With respect to the slopes, there were some differences, particularly for the shoulder. Thus, the psychophysically measured comfort functions appeared to have some relation to the hypothetical cost functions discussed here, but the two were probably not identical.

If the minimum cost principle is a good description of the results, the position of the arm should depend only on the joint angles and not on other properties of the arm geometry, such as the length of the limb. To test this prediction, we repeated the experiments, using two pointers of different lengths. To directly compare the results of the different experiments, we applied the following procedure: The subject performed movements to 15 target points, using the standard pointer with a length of $28 \mathrm{~cm}$. In the second session, the pointer was extended to $56 \mathrm{~cm}$ and 15 new target points were presented. These new target points were selected separately for each subject in the following way: For the arm position assumed at each original target point, the line of the hand was extended by $28 \mathrm{~cm}$, and the end of this line was used as the new target point. In this way, the joint angles assumed using the standard and the extended pointers should be equal for each pair of original

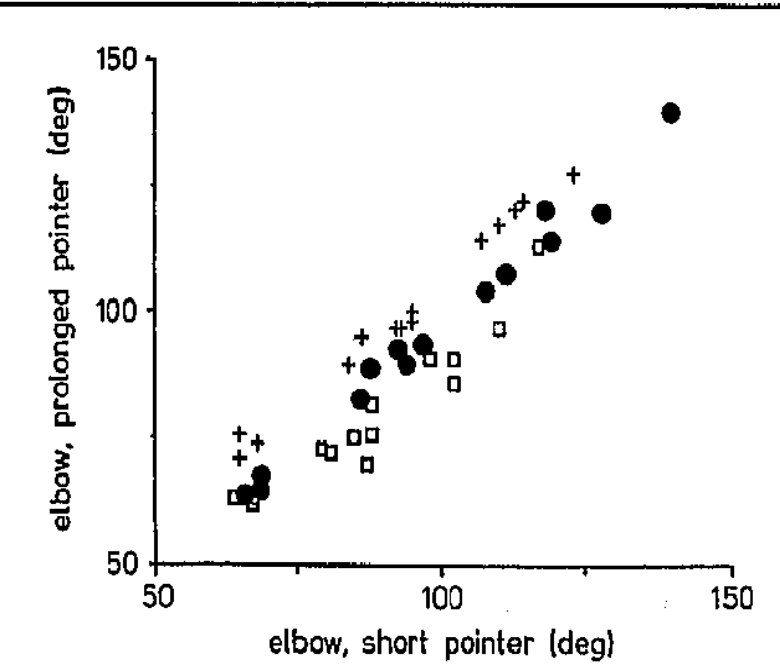

FIGURE 3. Comparison of the elbow angles when the distal limb of the three-joint arm was short or long. The three symbols represent the results of three different subjects. Each symbol shows the results of two individual measurements (Cruse et al., 1990).

\begin{tabular}{|lcc|}
\hline \multicolumn{2}{|c|}{$\begin{array}{c}\text { TABLE 1 } \\
\text { Mean } \begin{array}{c}\text { Deviation of Elbow Angle (Degrees) } \\
\text { When Loaded by a Spring }\end{array}\end{array}$} \\
\hline \hline Subject & Mean & \pm Standard deviation \\
\hline 1 & 9.7 & 3.6 \\
2 & 7.4 & 5.1 \\
3 & 15.3 & 7.0 \\
4 & 8.4 & 6.2 \\
5 & 8.6 & 3.2 \\
6 & 7.4 & 3.2 \\
7 & 8.9 & 5.9 \\
8 & 13.2 & 9.7 \\
\hline$N=20$. All deviations were significant $(p<.01)$. \\
\hline
\end{tabular}

and new target points if the change in the limb's length does not influence the form of the cost functions.

The results for three subjects are shown in Figure 3. Each dot represents the value of the elbow angle for a pair of targets with the standard pointer (abscissa) and the long pointer (ordinate). The strong correlation showed that the length of the distal limb had no significant effect on the values of the selected angles although it considerably changed the geometry of the whole arm.

At least two interpretations of the cost functions are possible: (a) They might represent the physiological costs necessary to maintain a given joint angle or (b) they might be completely independent of any kind of physiological costs and merely represent a fixed "computational" value used by the neural system to solve the redundancy problem. Applying a load or external force to the arm changes the physiological costs. Therefore, according to the first interpretation, different joint angles should be adopted in pointing to a given target position when the elbow joint is loaded. According to the second assumption, no differences should occur. These predictions were tested experimentally by mounting a spring across the elbow joint to apply a force at the elbow that depended on the joint angle. The force increased approximately linearly from zero at an elbow angle of $125^{\circ}$ to $2,300 \mathrm{~g}$ at an elbow angle of $30^{\circ}$. All eight subjects investigated showed a highly significant deviation of selected elbow angles in the direction of the applied force (Table 1). This result supported the first interpretation: The hypothetical cost functions seem to be related to the physiological costs necessary to hold the joint in a given position.

\section{Two Network Models That Solve the Static Problem}

In the model calculation just described, the cost functions were represented explicitly by parabolic functions. The question arises as to how these cost functions might be realized in the brain. Two simulations (Brüwer \& Cruse, 1990) showed that implicit cost functions can be "learned" 
by simple distributed neural networks. One model consisted of a three-layer artificial neural network with only feedforward connections. The activities of the two input neurons corresponded to the values of the cartesian workspace coordinates $x$ and $y$. The output layer contained three units. Their activities corresponded to the values of the three joint angles. The network was trained by using the arm configurations of one subject pointing to 32 target points. With an intermediate layer of 20 "hidden" units, the network could generalize with sufficient exactness (mean error of $0.9 \mathrm{~cm}$ ) to untrained targets.

In the second network simulation, the output layer again consisted of three neurons, and their activities represented the values of the three joint angles. The input layer con-

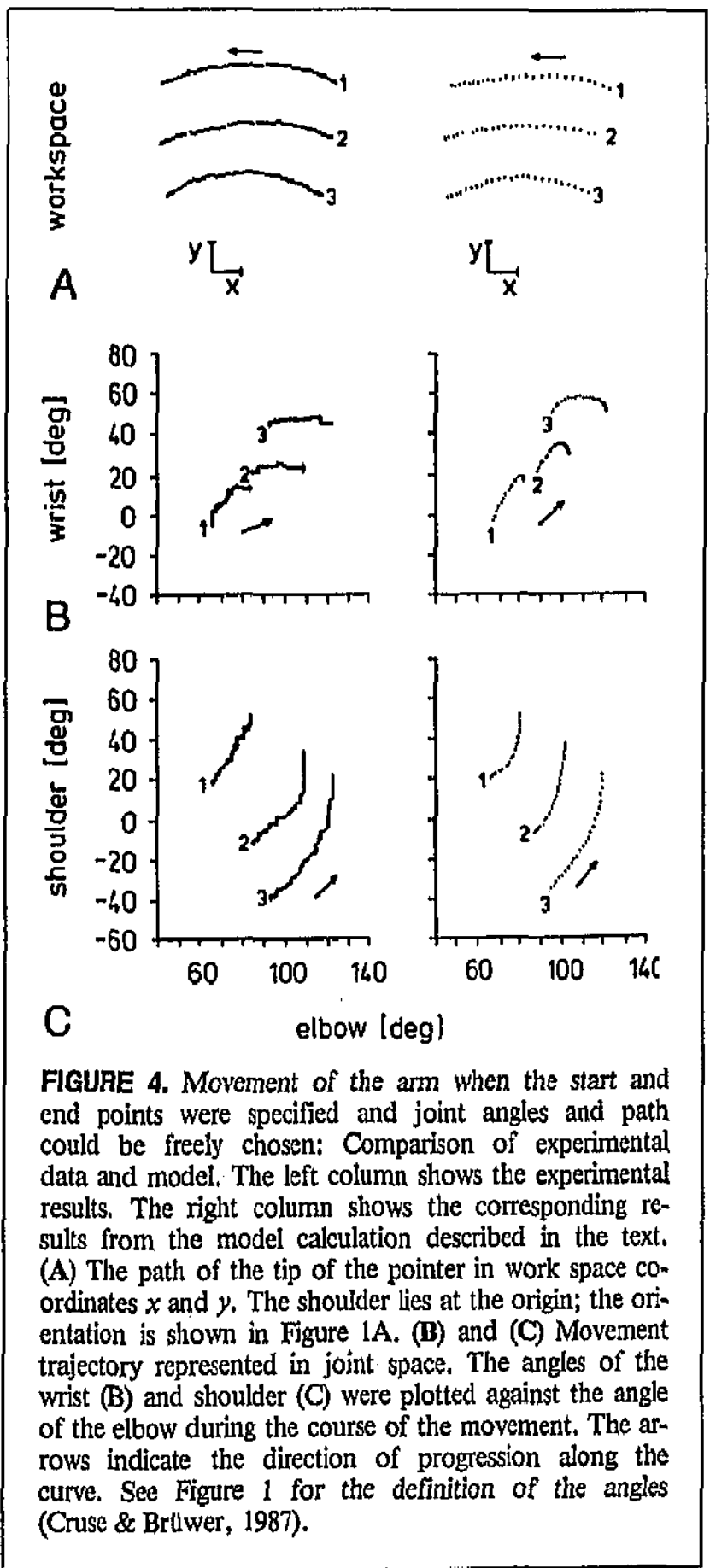

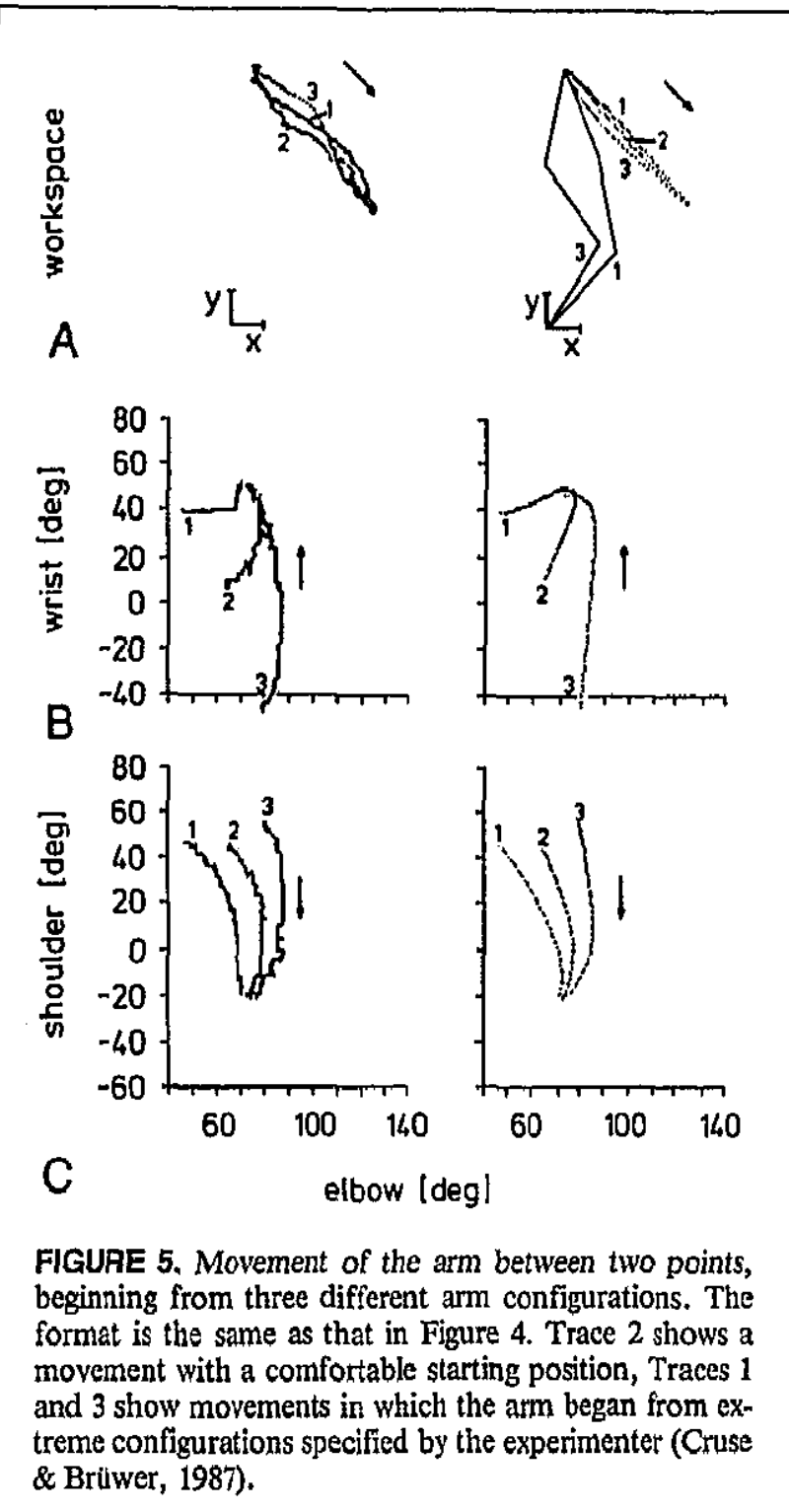

sisted of a two-dimensional array of $10 \times 10$ neurons, which represented the two-dimensional work space. These input units acted like a kind of simple retina with overlap between the receptive fields. The target position was coded in the pattern of activity in the input layer. Like the first model, this network also was able to reproduce trained and untrained arm configurations with good accuracy (mean error of $1.4 \mathrm{~cm}$ ). In future studies, these models will be used as one module in an extended neural network model that will also include other aspects necessary to simulate the kinematic behavior.

\section{Kinematic Control: Selecting Configurations to Perform a Movement to a Target}

In the preceding experiments, only the starting point and the end point for the tip of the pointer were specified. The subject was free to choose any trajectory for the movement, and only the final configuration of the arm was analyzed. In the following experiments, the kinematics of the movement itself were considered. The questions investigated 
were the following: What kinds of paths are adopted? Is it possible to say something about the mechanisms that produce these paths? In particular, does the minimum cost principle apply?

The subjects were asked to make the movements in a comfortable way. In particular, they were asked to move slowly rather than quickly. Movement durations typically were between 1.5 and $2 \mathrm{~s}$. The slow speed allowed us to neglect the dynamic effects, for example, inertial forces, as a first approximation. Selected results are shown in Figures 4 and 5 (for further results, see Cruse \& Brüwer, 1987). For one set of movements, the lines in the work space connecting starting points and targets were parallel to the $x$ axis. For these movements, the paths of the pointer in work-space coordinates were nearly straight lines in most cases, but curved paths sometimes occurred (e.g., Figure 4). The paths in joint space were generally curved, but joint movements were monotonic. For the second set of movements, the lines connecting the starting points and targets formed an angle of $45^{\circ}$ relative to the work-space coordinates (Figure 5). Unlike the experiments shown in Figure 4 , the wrist angle at the start was placed by the experimenter in an extreme and rather uncomfortable configuration at the start. Under these conditions, several movements involved nonmonotonic changes in joint angles, that is, joint movements reversed direction during the movement (e.g., Figure 5, Trace 3 for the elbow joint).

The other significant result presented by Cruse and Brüwer (1987) was that the path depended both on the joint angles at the start and on the position of the target. One might argue that a local control principle based merely on the direction of the end point, rather than on its absolute position, might be sufficient. The curved paths, however, showed that both the direction and the distance between the two points affected the form of the path. Paths from a given starting point to targets that lay at different distances in the same direction differed from the beginning (not shown). This means that the path reflects both global and local control laws.

\section{A Kinematic Model}

As the experimental results showed, the paths of the end effector usually formed straight lines in the work space, but under some conditions they were curved. The model that was developed to explain these results contains two parts. The experimental results showed that the path for a comfortable movement between two points most often approximated a straight line of the end effector in the work space. Therefore, the first part is a model that is constrained to produce straight paths in work space. Based on this requirement, the model determines the movements required for the joints. For a nonredundant system, the joint angles are completely determined by geometrical constraints once the form of the path is fixed.

For a redundant system the selection is more complicated. One might postulate a mechanism based on minimiz- ing the total cost value, that the arm simply assumes the configuration with lowest cost at each position along the straight path. As described earlier, the minimum of the cost function for each joint occurred at angles near the middle of the range. Thus, according to this hypothesis, the curves in the joint space coordinates should always tend to pass through the points corresponding to these cost minima. The results showed that this was not the case.

Moreover, the minimum cost principle leads to the prediction for the start from an uncomfortable arm position that joints should immediately move to a comfortable position without moving the tip of the end effector and then continue to move the end effector along the straight path. The actual arm, however, moved the tip of the end effector immediately and seemed to approach angle values that correspond to the minimuin cost principle only gradually during the ongoing movement (Figure 5).

Thus the minimum cost principle alone is not able to describe the experimental results. Another mechanism is needed. To aid the following discussion, Figure 6 shows the

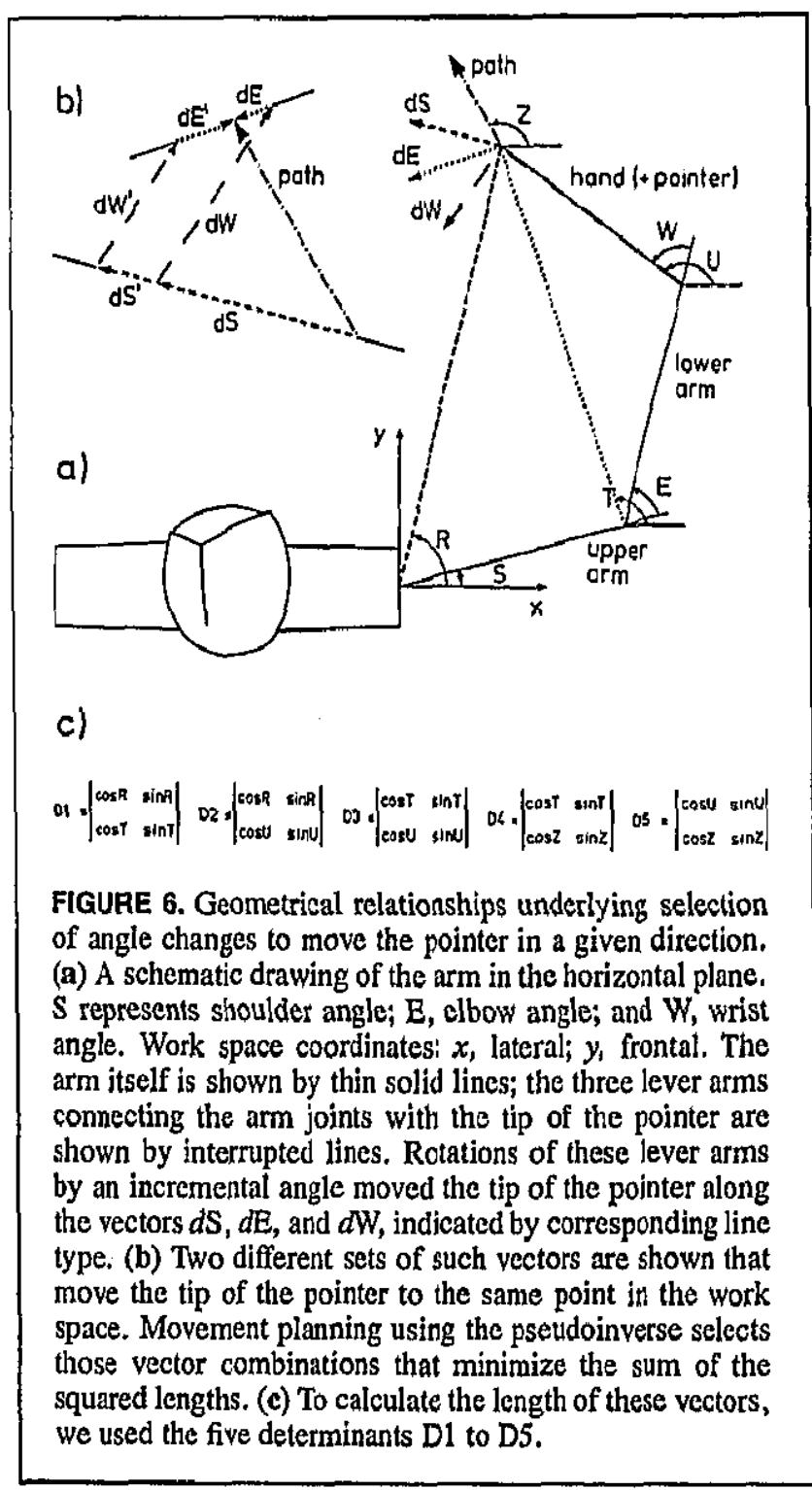


three lever arms that connect each joint with the end effector, that is, the tip of the pointer, and the three vectors that describe the movement of the end effector as the result of incremental changes in the angle at each of the three joints.

One hypothesis often proposed for controlling the movement of a redundant manipulator is the so-called pseudo. inverse or Moore-Penrose generalized inverse (e.g., Hollerbach \& Suh, 1985). This method solves the redundancy problem in the following way: The incremental angle changes are calculated so that the sum of the squared vector lengths $d \mathrm{~S}, d \mathrm{E}$, and $d \mathrm{~W}$ (see Figure 6) is a minimum. This means that all three vectors have, as far as geometrically possible, about the same length, or, in other words, that the movement is distributed over all three joints in an economical manner. As this seems to correspond to the experimental result described earlier, a model calculation was attempted that applied the pseudoinverse control to produce a straight path in the work space. According to this method, the vector lengths are given by the following Equations $1-3$ ( $f=0$; for definition of D1 to D5, see Figure 6):

$$
\begin{aligned}
& d S=\frac{-(\mathrm{D} 2 * \mathrm{D} 5+\mathrm{D} 1 * \mathrm{D} 4) / \mathrm{D} 3^{2}-\mathrm{f}^{*} \mathrm{~K}}{\left(\mathrm{D} 1^{2}+\mathrm{D} 2^{2}\right) / \mathrm{D} 3^{2}+1} . \\
& d \mathrm{E}=-d \mathrm{~S}^{*} \mathrm{D} 2 / \mathrm{D} 3-\mathrm{D} 5 / \mathrm{D} 3 . \\
& d \mathrm{~W}=d S * \mathrm{D} 1 / \mathrm{D} 3+\mathrm{D} 4 / \mathrm{D} 3 .
\end{aligned}
$$

The simulations of the pseudoinverse control showed that many movements could be at least qualitatively described. In some cases, however, obvious qualitative disagreements between experimental data and the predictions of the model were present. Thus, the pseudoinverse control alone was also unable to describe the results. The discrepancy can be described in the following way: In some movements, the simulation specified that two or more joints were to move to more extreme angles, whereas the real arm never does this, even when the alternative requires a larger movement in the joints. This behavior suggests that one rule is to avoid unnecessary movements toward extreme joint angles. This corresponds to the minimum cost principle mentioned earlier.

Because neither the minimum cost hypothesis nor the pseudoinverse hypothesis alone was able to describe the experimental results, a combination of both was tried. For this simulation, the vector lengths were calculated according to the pseudoinverse rule (Equations 1-3). Then, the vector lengths were weighted by the value of the corresponding cost functions. This was done by using an appropriate value for $f$ in Equation 1. This factor weights the contribution, $K$, of the cost functions whereby $\mathrm{K}$ is the sum of the costs for the angles at the three joints. The combination of these two "Iocal" control rules-the pseudoinverse control and the minimum cost principle-provided quite reasonable descriptions of those movements that showed a straight path.

Curved paths, however, appeared under some conditions in the experiment. What might have caused these deviations

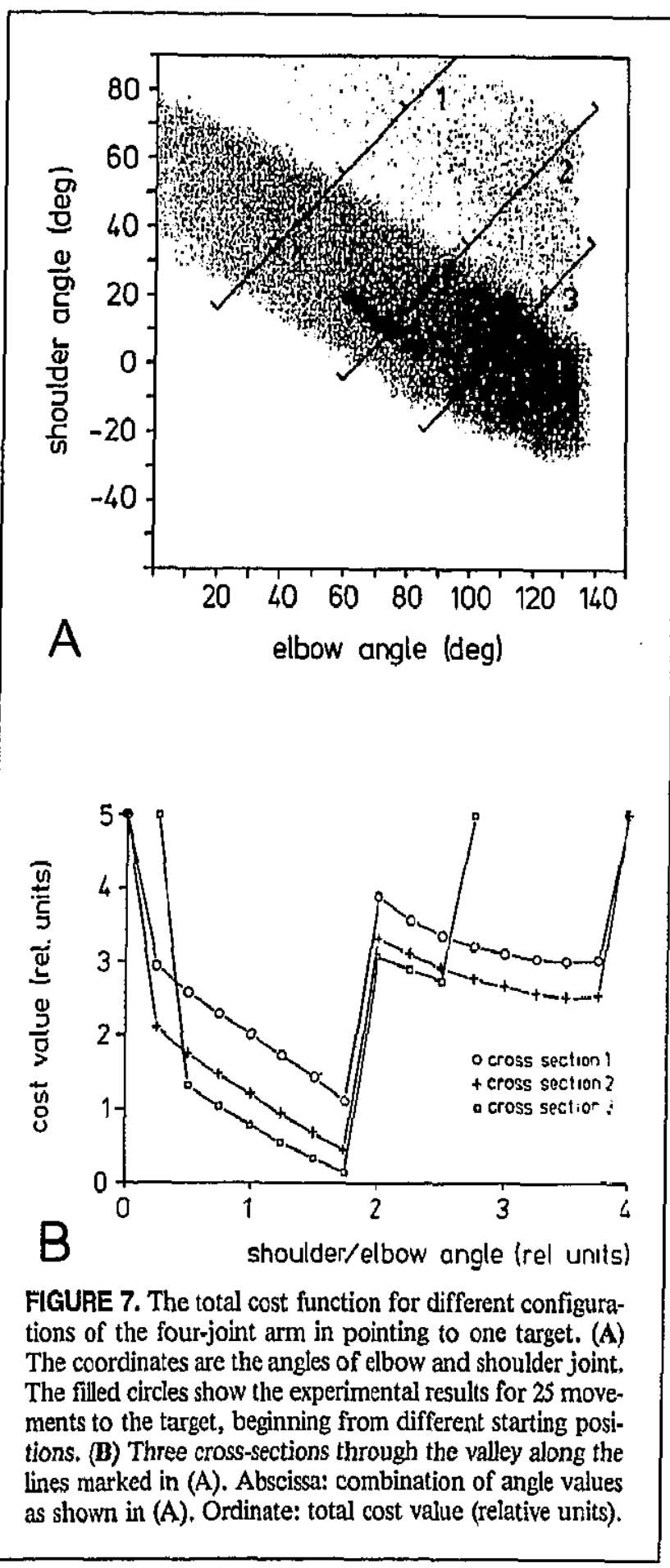

from the straight paths? A third hypotheses could provide a possible explanation. The mass-spring hypothesis (Bizzi, Hogan, Mussa-Ivaldi, \& Giszter, in press; Feldman, 1986) or end-point control hypothesis assumes that the values of the joint angles of the final position are calculated before the movement has started. The movement is performed by letting each joint move to its final angle independently of the other joints. Although this "global strategy" is an appealing hypotheses with several theoretical advantages (see Hollerbach \& Atkeson, 1987), it was not supported by the results. According to this hypothesis, one should find nearly straight lines in the joint space presentation, and 
nonmonotonic trajectories in joint space should be excluded. Because such reversals did occur, this hypothesis can be ruled out as a sole explanation.

Curved paths seemed to appear for those movements in which a straight path would have required strongly nonlinear trajectories in the joint space. This suggests that the end-point control was actually used in these cases. Thus the control system might use a compromise between a straight line in the joint space and a straight line in the work space. Such a compromise was implemented in a two-part model in the following way: The incremental angle changes calculated using the local strategies described above were added to changes that corresponded to the end-point control hypothesis. At the beginning of the movement, global and local strategies contributed equally to elbow and wrist movement, whereas the ratio was 2:1 for the shoulder joint. Because the mass-spring control signal depends on the difference between the actual angle and the desired or final joint angle, the contribution of the global strategy decreases linearly to zero through the course of the movement. Therefore, an accurate estimate of the joint angles for the final arm position, which is a necessary assumption in the pure mass-spring hypothesis, is not critical. Approximate values suffice. This compromise can produce deviations from the straight paths in the work space and decrease the curvature of the trajectories in the joint space. The results of the model calculation are shown in Figures 4 and 5 together with the experimental results to allow direct comparison. A more detailed description was given in Cruse and Brüwer (1987) and Cruse (1989).

\section{Control of a Four-Joint Arm: Resting Position}

The minimum cost principle provided a sufficient description of the resting arm configurations for the threejoint arm, one with superfluous degrees of freedom. Therefore, this principle was extended to an arm with four joints, one with two superfluous degrees of freedom. For the corresponding experiments, the manipulandum was augmented by a fourth joint and a fourth limb segment with a length about equal to those of the other three. The additional joint was moved by the fingers, using a lever arm and pulley (Cruse et al., 1990).

Application of the minimum cost principle to the fourjoint arm with two degrees of freedom did not provide an adequate description of the experimental data. Because of this disappointing result, we used a model calculation to analyze the total cost for all possible arm configurations for a given target point. The result of these calculations showed that the total cost function forms a valley with quite steep walls, but this valley can be very long and contain a large fraction of the geometrically possible configurations. This is shown in Figure 7a for one particular target point. The cost is represented by the gray shading: High density corresponds to low total cost values. The white areas represent angle combinations for which the target cannot be reached. The occurrence of such valleys for a given target means that many configurations of the four-joint arm have nearly the same total cost.

Thus, even if the control system uses the minimum cost principle, considerable variation in the arm configurations selected for a given target is to be expected. This prediction was confirmed experimentally by analyzing movements from 15 different starting positions to one target. The standard deviation of the final angles was measured for all four joints. The results showed larger standard deviations for the four-joint arm than for the three-joint arm. According to the results shown in Figure 7, the distribution of the final joint configurations should not be random but rather should be concentrated within the valley of minimum cost. This prediction was confirmed by superimposing the experimental results (filled circles in Figure 7a) on the calculated cost function. This agreement supports the hypothesis that the minimum cost principle applies also to the control of the four-joint arm. The distribution of measured configurations was somewhat broader than that of the absolute theoretical minimum, however. In part, this scatter was due to significant correlations between the final angle of a joint and its angle at the beginning of the movement. This is shown in Figure 8 for one subject and one target point. This result means that the final configuration depends on the initial

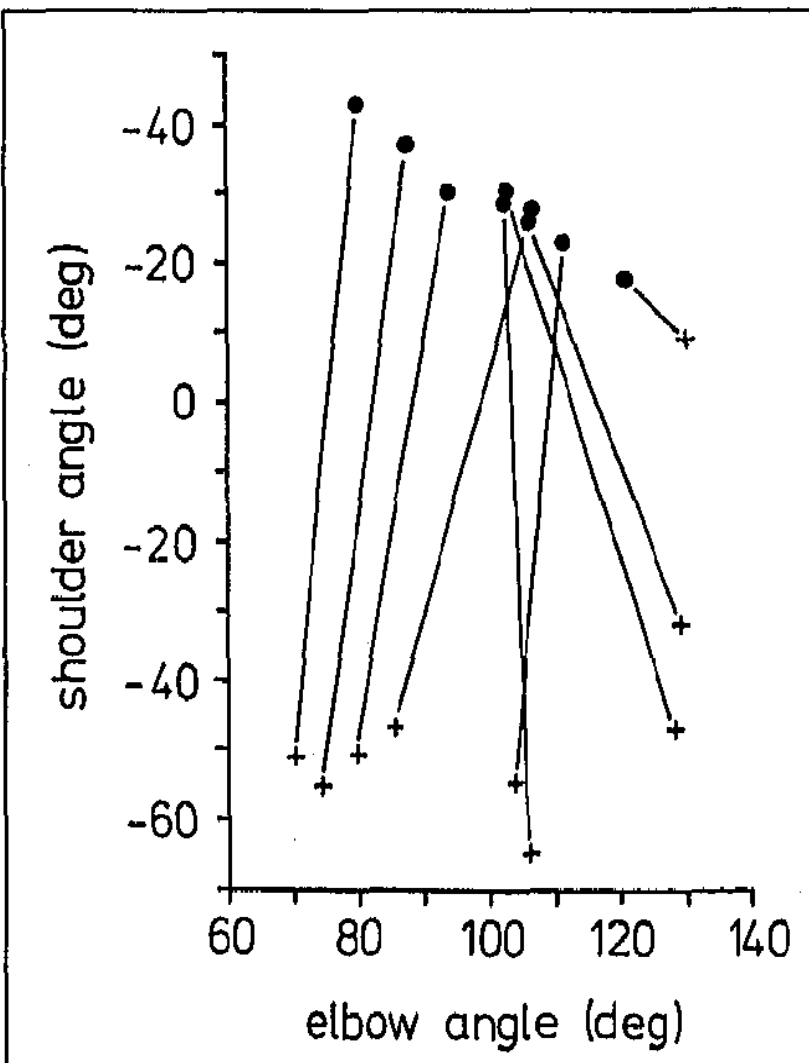

FIGURE 8, The dependence of the final joint angles on the initipl angles for target movement with the four-joint arm. Corresponding initial (crosses) and final positions (circles) are connected by thin lines. For each starting position, the arm pointed to a different point, whereas all final positions corresponded to the same target point. The target was the same as that used in Figure 7. The subject was different. 
configuration. Thus, the minimum cost principle, in essence, appears to apply to the descriptions of the four-joint arm as well as the three-joint arm, but for the former this mechanism must be supplemented by additional mechanisms to account for the influence of the prior configurations or movements. Such a dependence is rarely evident for movements that use the three-joint arm (see Figure 5 for one example).

\section{Conclusions}

In summary, the experimental results and simulations presented here suggest that the control of arm movements involves a compromise among four requirements: (a) optimal distribution of the movement among all joints (pseudoinverse), (b) minimization of the static costs defined by the cost functions (minimum cost principle), (c) minimization of the inertial forces acting at the tip of the end effector perpendicular to the direction of movement (by following a straight path in the work space), and (d) simplification of the pattern of muscle activity (by avoiding nonmonotonic joint movements, as far as possible). Two points should be added with respect to (c). First, a preference for straightline trajectories has been described in many previous studies (e.g., Abend, Bizzi, \& Morasso, 1982; Morasso, 1981). Second, minimizing inertial forces-both perpendicular to and parallel to the movement direction-at the end effector is the central feature of the minimum-jerk model (Flash \& Hogan, 1985; Hogan, 1984, 1988), which can explain many temporal and spatial aspects of movement trajectoties. The final requirement (d) reflects a measure of end" point control (Bizzi et al., in press). Thus, movement planning even in the simplest case, that of pointing movements, appears to reflect several different organizing principles. Most explanatory models have focused exclusively on one or another of these. We feel the search for a single best principle does not do justice to the complexity and adaptability of the motor system. Moreover, the number of factors is apt to increase when more complex situations are studied. Our current research on obstacle avoidance is confirming this suggestion.

\section{REFERENCES}

Abend, W., Bizzi, E., \& Morasso, P. (1982). Human arm trajectory formation. Brain, 105, 331-348.

Bizzi, E., Hogan, N., Mussa-Ivaldi, F. A., \& Giszter, S. (in press). Does the nervous system use equilibrium-point control to guide single and multiple joint movement? Behavioral Brain Science.

Brüwer, M., \& Cruse, H. (1990). A network model for the control of the movement of a redundant manipulator. Biological Cyber. netics, 62, 549-555.

Cruse, H. (1986). Constraints for joint angle control of the human arm. Biological Cybernetics, 54, 125-132.

Cruse, H. (1989). The control of path and joint angles in a human arm. In L. Personnaz \& G. Dreyfus (Eds.), Neural networks from models to applications (pp. 71-77). Paris: I.D.S.E.T.

Cruse, H., \& Brilwer, M. (1987). The human arm as a redundant manipulator: The control of path and joint angles. Biological Cybernetics, 57, 137-144.

Cruse, H., Wischmeyer, E., Brüwer, M., Brockfeld, P., \& Dress, A. (1990). On the cost functions for the control of the human arm movement. Biological Cybernetics, 62, 519-528.

Feldman, A. G. (1986). Once more on the equilibrium-point bypothesis $(\lambda$ model) for motor control. Journal of Moior Behavior, 18, 17-54.

Flash, T., \& Hogan, N. (1985). The coordination of arm movements: An experimentally confirmed mathematical model. Journal of Neuroscience, 5, 1688-1703.

Hogan, N. (1984). An organizing principle for a class of voluntary movements. Journal of Neuroscience, 4, 2745-2754.

Hogan, N. (1988). Planning and execution of multijoint movements. Canadian Journal of Physiological Pharmacology, 66, 508-517.

Hollerbach, J. M., \& Atkeson, C. G. (1987). Deducing planning variables from experimental arm trajectories: Pitfalls and possibilities, Biological Cybernetics, 56, 279-292.

Hollerbach, J. M., \& Suh, K. C. (1985). Redundancy resolution of manipulators through torque optimization, Proceedings of the 1985 IEEE International Conference on Robotics and Automation. Silver Spring, MD: IEEE Computer Society Press.

Kitkpatrick, S., Gelatt, C. D., \& Vecchi, M. P. (1983). Optimization by simulated annealing. Science, 220, 671-680.

Morasso, P. (1981). Spatial control of arm movements. Experimental Brain Research, 42, 223-227.

Rosenbaum, D., Slotta, J. D., Vaughan, J., \& Plamondon, R. (1991). Optimal movement selection. Psychological Science, 2, 86-91.

Submitted September 9, 1991

Revised June 6, 1992 